\title{
CELE Échanges, Centre pour des environnements pédagogiques efficaces 2009/09
}

\section{Évaluer la qualité}

des espaces éducatifs :

\section{Etude pilote du CELE/ \\ OCDE}

\section{Hannah von Ahlefeld}

https://dx.doi.org/10.1787/220431820773 


\section{Évaluer la qualité des espaces éducatifs : étude pilote du CELE/OCDE}

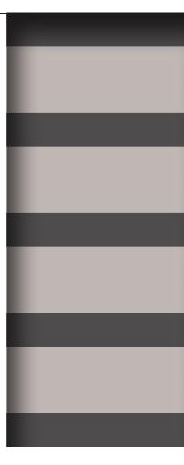

Par Hannah von Ahlefeld, OCDE

L'Étude pilote internationale du CELE pour l'évaluation de la qualité des espaces éducatifs a pour objectif d'aider les autorités éducatives, les écoles et d'autres à maximiser l'utilisation de et les investissements dans les environnements pédagogiques. Cet article donne un aperçu de l'avancement de l'étude pilote, qui est actuellement mise en œuvre au Brésil, au Mexique, en Nouvelle-Zélande, au Portugal et au Royaume-Uni.

\section{CONTEXTE}

Le Centre pour des environnements pédagogiques efficaces (CELE) de I'OCDE a commencé à travailler sur l'évaluation de la qualité des équipements éducatifs en 2005 à l'occasion de deux réunions d'experts internationaux sur le sujet à Lisbonne, Portugal, et Telchac-Puerto, Mexique (voir www.oecd.org/edu/ equipements/evaluationqualite). Ces réunions étaient organisées en réponse à l'intérêt croissant $d^{\prime} u n$ certain nombre de membres du CELE pour un thème, qui intéresse de plus en plus la recherche et devient une priorité politique : à savoir, l'importance de communiquer avec les utilisateurs des environnements pédagogiques et les autres parties prenantes pour mieux comprendre comment, et avec quelle efficacité, les environnements viennent appuyer les objectifs éducatifs et autres.

Définir la qualité dans le contexte de systèmes d'éducation est un challenge. Le Cadre du CELE pour l'évaluation de la qualité des espaces éducatifs a pour but de définir la qualité dans le domaine des politiques éducatives, et selon une série de principes et critères. II considère également le processus d'évaluation, le stade auquel il convient d'évaluer une installation au cours de son cycle de vie, les parties prenantes et le(s) outil(s) utilisé(s). Le cadre « met en évidence les corrélations, au cours du cycle de vie d'un bâtiment scolaire, entre les grandes orientations qui interviennent dans les questions liées à la qualité des équipements éducatifs ; de déterminer les positions actuelles sur ce qui définit "la qualité" des équipements; d'analyser les attentes et les avantages associés aux installations éducatives pour leurs multiples utilisateurs et parties prenantes; et d'étudier les méthodes appropriées pour évaluer différents aspects de la qualité. »

Le cadre constitue un modèle conceptuel pour l'essentiel des travaux du CELE qui ont suivi dans ce domaine, notamment l'Étude pilote internationale d'évaluation des performances des espaces éducatifs (EQES). Néanmoins, le cadre continuera d'évoluer, notamment au vue des résultats de cette étude pilote.

EQES a été lancée début 2007, initialement en tant qu'Étude pilote de l'OCDE sur l'évaluation des performances des établissements. 


\section{LA PROPOSITION DE L'EQES}

La méthodologie de l'étude EQES s'inspire des évaluations après emménagement, des évaluations sur les performances des bâtiments et de la recherche plus récente sur les études d'utilisabilité, qui ont pour but d'évaluer de manière systématique les performances et/ou l'efficacité d'un ou plusieurs aspects d'un espace éducatif rapportés à un large éventail de questions en matière d'espace ou autre. Les outils de recherche, tels que les questionnaires adressés aux parties prenantes, les parcours scéniques, les groupes de discussion et I'observation, sont traditionnellement utilisés dans ce type d'étude. L'étude pilote EQES se fonde également sur la recherche menée sur le rôle de l'espace éducatif en tant qu'outil d'adaptation aux besoins et aux exigences changeants des programmes scolaires et de la pédagogie.

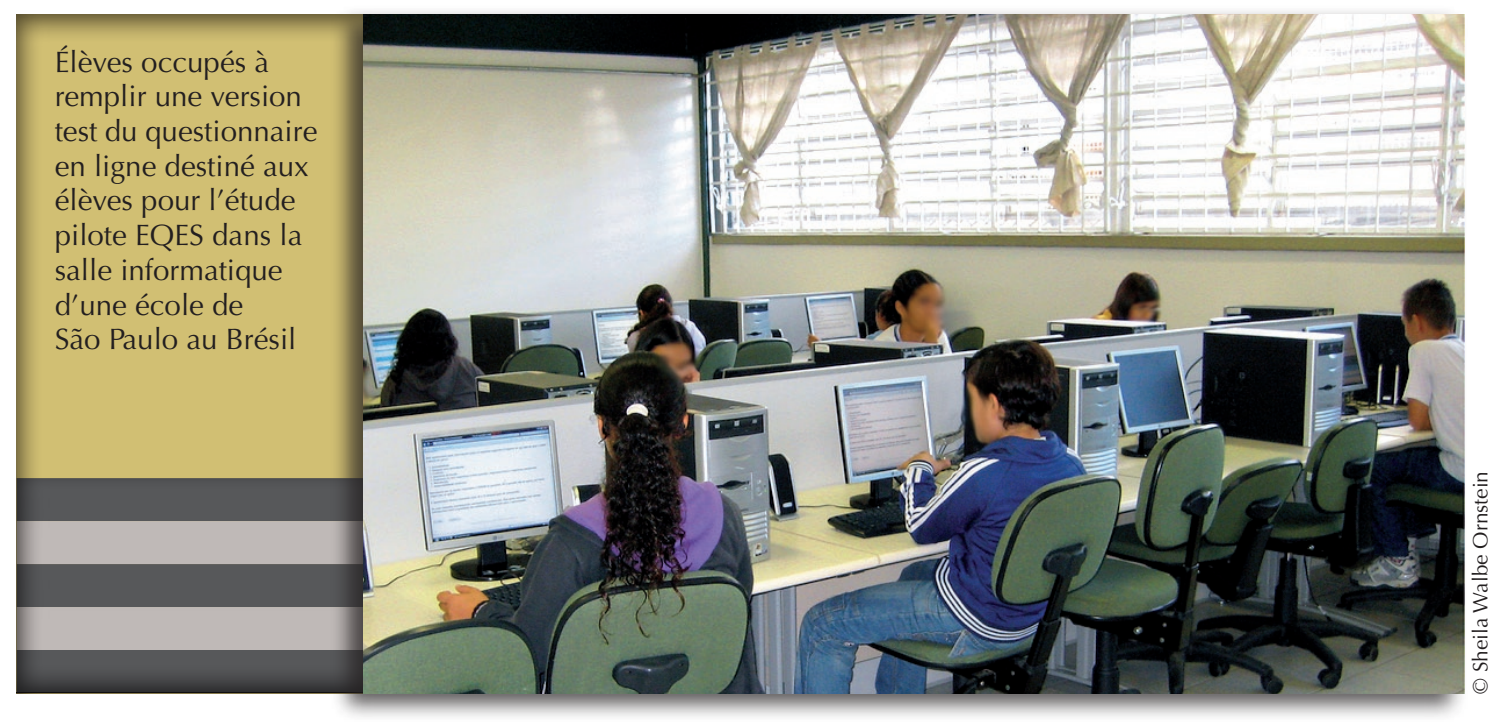

Les écoles participant à cette étude pilote seront évaluées selon deux principaux critères en matière de politique éducative identifiés dans le Cadre du CELE pour l'évaluation de la qualité des espaces éducatifs :

- La capacité de l'espace à accroître l'accès et l'égalité de tous face à l'éducation. L'espace fournit un accès équitable à l'enseignement, en offrant une capacité spatiale adaptée à la demande. Cette considération reconnaît le droit fondamental de tous les individus à avoir accès à un établissement d'enseignement. Elle traite également des problèmes de surpopulation, qui peuvent compromettre le confort et la sécurité des utilisateurs des bâtiments, et des problèmes de taux de remplissage insuffisants, qui peuvent avoir un impact négatif sur l'état d'âme de l'école.

- La capacité de l'espace à améliorer l'efficacité de l'enseignement et à encourager l'acquisition de compétences clés. L'espace est au service de programmes d'apprentissage et de pédagogies variés. II facilite l'interaction des individus au sein de groupes socialement hétérogènes; il fournit aux individus les armes leur permettant de donner un sens à leur vie et de gérer leur vie de façon responsable en exerçant un contrôle sur leur environnement pédagogique ; et il offre un environnement dans lequel les élèves sont encouragés à utiliser divers outils de façon interactive.

L'étude pilote a trois objectifs :

- Développer des outils faciles à utiliser et efficaces, ainsi que des stratégies de collecte de données, en utilisant des méthodologies définies d'un commun accord. Il importe que les résultats de ces évaluations soit utilisés, afin d'optimiser l'efficacité éducative de ces espaces. De plus, le développement et l'essai d'outils permettront de valider l'approche utilisée dans l'étude globale. 
- Identifier les bonnes pratiques et les « leçons apprises » dans les pays participants, en vue d'améliorer la qualité des espaces éducatifs au cours du cycle de vie des bâtiments.

- Étudier les problèmes et contraintes contextuels afin d'améliorer la qualité des espaces éducatifs et d'établir des références générales par rapport auxquelles la performance de l'établissement pourra être évaluée.

\section{PAYS PARTICIPANTS ET SÉLECTION DES ÉCOLES}

Pour faciliter la gestion du projet, un petit nombre d'écoles sélectionnées dans un nombre limité de pays ont participé à l'étude : entre un et cinq établissements secondaires (ISCED 2) au Brésil, au Mexique, en Nouvelle-Zélande, au Portugal et au Royaume-Uni (Angleterre et Écosse). L'objectif n'est pas de mener des comparaisons quantitatives entre les pays.

Chaque pays participant a désigné un coordinateur national (ou contact principal) pour l'étude. Dans la plupart des pays, une équipe de recherche plus grande constituée de membres provenant d'universités et d'autres établissements - tels que I'Université de Sao Paulo (Brésil), I'Université de Salford (Angleterre), I'Université de Monterrey (Mexique), I'IST (Portugal), et Architecture and Design Scotland - a été formée, afin d'assister les coordinateurs nationaux dans la gestion de projet et l'analyse de données.

\section{Tableau 1. Nombre d'écoles sélectionnées par pays pour participer à l'EQES}

\begin{tabular}{|ll|}
\hline Brésil & 1 \\
\hline Mexique & 5 \\
\hline Nouvelle-Zélande & 2 \\
\hline Portugal & 4 \\
\hline Royaume-Uni : Angleterre & 2 \\
\hline Royaume-Uni : Écosse & 6 \\
\hline
\end{tabular}

Les coordinateurs nationaux ont utilisé les critères suivants afin d'identifier les écoles qui participeront au projet :

- l'évaluation d'une école récemment construite dont pourrait s'inspirer la conception des futures écoles ;

- l'évaluation d'une école existante afin de définir les avantages d'une rénovation du bâtiment versus I'abandon de la totalité ou d'une partie importante du bâtiment ;

- l'évaluation de l'efficacité d'un bâtiment récemment remanié ou agrandi ;

- l'évaluation du processus de planification et de conception en vue d'améliorer la qualité de I'enseignement dans une école.

Les pays ont également utilisés des critères tels que la taille de l'école, le contexte démographique (zones rurales vs. zones urbaines) et le contexte socio-économique pour sélectionner les écoles.

\section{DÉVELOPPEMENT DU MANUEL EQES : UN EFFORT DE COLLABORATION}

Un groupe d'experts internationaux a été formé pour travailler avec le Secrétariat du CELE sur la rédaction d'un manuel pour le projet; le manuel de 70 pages a été finalisé début 2009.

Le principal objectif du manuel est de fournir un guide pratique et facile à utiliser à ceux qui sont impliqués dans l'étude pilote EQES : coordinateurs nationaux et équipes de recherche, personnel 
enseignant, élèves et directeurs d'établissements, entre autres. Il comprend les quatre outils suivants, que les pays sont tenus d'utiliser :

- Le classement prioritaire des objectifs de qualité de I'OCDE. Cet outil a pour objectifs 1) de mieux comprendre les problèmes et contraintes contextuels plus larges qui pourraient avoir un impact sur la qualité des espaces éducatifs, et 2) d'établir les références par rapport auxquelles la performance de l'école pourra être évaluée. Vingt-deux objectifs de qualité OCDE ont été développés, chacun reflétant globalement les critères définis dans le Cadre d'évaluation du CELE.

- L'étude de l'installation éducative. Cet outil a pour objectifs 1) de fournir des informations descriptives sur l'école, en vue de définir le contexte social, économique, démographique, éducatif et opérationnel dans lequel les objectifs de performance des écoles en termes de qualité seront établis, et 2) de recueillir des données comparatives auprès du personnel et des étudiants sur les aspects objectifs - ou quantifiables - liés à la qualité des espaces éducatifs.

- Questionnaires destinés aux élèves et au personnel. Cet outil a pour objectifs 1) de mieux comprendre comment le personnel et les élèves perçoivent la qualité des espaces éducatifs en termes d'accessibilité, d'utilisation de l'enseignement et des espaces pédagogiques, de confort, d'image de l'école, de sûreté et de sécurité, et de maintenance, et 2) de collecter des données comparatives auprès du personnel et des élèves sur les aspects subjectifs liés à la qualité des espaces éducatifs. Des questionnaires en ligne sont mis à disposition pour la collecte de ces données.

- Groupes de discussion. Cet outil a pour objectifs 1) d'étudier de façon plus approfondie les questions communes et contradictoires soulevées dans les questionnaires destinés aux élèves et au personnel enseignant, et 2) de développer des recommandations communes (dans le cadre de groupes de discussion composés d'élèves et de personnel enseignant) sur l'amélioration des espaces éducatifs de l'école. Chaque école est tenue de mettre en place au moins un groupe de discussion composé d'élèves et un groupe animé par le personnel enseignant.

Pour chaque outil, le manuel présente les objectifs, les questions de recherche, la durée prévue et réelle de réponse, les instructions étape par étape sur la manière de mettre en place l'outil (y compris les exigences minimum), et il fournit une présentation sur la manière dont les résultats apparaîtront dans le rapport final. Des modèles de questionnaires, de rapports d'activité et de comptes rendus post-projet sont fournis dans les annexes du manuel.

Chaque outil de recherche possède un certain degré de flexibilité qui permet aux pays de l'adapter aux conditions locales et aux questions qui leur sont spécifiques d'un point de vue national et local, et à l'échelle des écoles. Les coordinateurs nationaux et les équipes de recherche peuvent donc modifier les outils en concertation avec le Secrétariat du CELE.

\section{LA PHASE DE MISE EN OEUVRE}

\section{Pré-test des questionnaires destinés aux élèves et aux enseignants}

En avril 2009, des collègues au Brésil et au Portugal ont testé avec succès les questionnaires en ligne destinés aux élèves et aux enseignants. Au Brésil, le directeur d'un établissement a demandé à 14 élèves âgés de 11 à 13 ans et à six enseignants de participer à l'activité. Le pré-test a été mené dans la salle informatique de l'école. Au Portugal, 12 élèves de 14 à 17 ans (secondaire) et un enseignant ont rempli le questionnaire. 
Les élèves et les enseignants ont rapporté que les éléments du questionnaire étaient pertinents et faciles à comprendre. Le temps nécessaire pour remplir les questionnaires est beaucoup plus court que les 30 minutes prévues, bien que la connexion Internet lente ait souvent ralenti le processus.

À l'issue de ce pré-test, un guide a été élaboré afin d'aider les élèves et les enseignants qui répondent aux questionnaires. Ce guide souligne par exemple l'importance de choisir le lieu le plus approprié pour le pré-test, de préparer à l'avance la salle où aura lieu le test et de s'assurer que les liens fonctionnent, de comprendre l'échelle de valeurs, de fournir entre autres un soutien technique aux personnes sondées durant le test, et de se doter d'un plan de secours en cas de problèmes techniques. Les traductions de certains éléments ont par ailleurs été modifiées.

\section{Questions relatives à la traduction et à la vérification}

En qualité de coordinateurs au Brésil, le Mexique et le Portugal ont commencé à organiser la traduction du manuel EQES. II est alors devenu évident qu'il était nécessaire d'établir des directives générales afin de les assister, et de mettre en place des équipes de projet pour la traduction et la vérification des matériaux. La traduction et la vérification sont un processus qui nécessite des ressources importantes, et bien qu'il n'ait pas été jugé nécessaire de traduire l'ensemble du manuel EQES de l'anglais vers la langue cible, il a été demandé aux pays de traduire l'ensemble des questionnaires et des rapports d'activité.

Au Brésil, au Mexique et au Portugal, un à deux traducteurs professionnels ont réalisé une traduction initiale, qui a ensuite été vérifiée par le coordinateur EQES et l'équipe de projet. Le coordinateur national s'est chargé de la vérification finale du manuel. Toutes les déviations par rapport à la version de la langue d'origine ont été méticuleusement consignées, notamment les ajouts, les suppressions, les contresens et les difficultés rencontrées avec la version anglaise. Certaines des difficultés rencontrées avec la version originale ont facilement été résolues, tandis que d'autres devront être traitées par l'ensemble des pays participants au terme de l'étude pilote.

Les quatre versions du manuel - en anglais, en portugais (Brésil et Portugal) et en espagnol - sont disponibles en ligne à I'adresse suivante: www.oecd.org/edu/facilities/evaluatingquality.

\section{Rapports d'activité, évaluation et questionnaires}

Il a été demandé aux coordinateurs nationaux de soumettre trois courts rapports d'activité durant la phase de mise en œuvre. Le rapport d'activité initial, que tous les pays ont rempli, demandait des informations sur les écoles sélectionnées, les contacts pour l'étude pilote, le calendrier de l'étude, l'utilisation des outils, les modifications apportées au manuel par le pays et les problèmes de traduction.

Dans leurs rapports initiaux, les pays ont signalé qu'ils prévoyaient d'achever l'évaluation des équipements éducatifs et les questionnaires destinés aux élèves et aux enseignants avant la fin 2009. Les pays soumettront leurs rapports d'activité finaux au Secrétariat du CELE d'ici à mars 2010.

De plus, certains pays ont ajouté plusieurs questions aux questionnaires destinés aux élèves et aux enseignants afin de mieux refléter les considérations et conditions locales. Par exemple, un pays a développé 40 questions supplémentaires pour les élèves, les enseignants et les directeurs d'établissements, afin d'aborder des questions spécifiques à leurs pays relatives au processus de planification et à l'amélioration de la qualité de la conception. De façon similaire, trois pays ont signalé qu'ils dirigeraient un groupe de discussion supplémentaire incluant des administrateurs ou d'autres utilisateurs du bâtiment scolaire. 


\section{RAPPORT FINAL ET TRAVAUX FUTURS}

Le rapport final de l'étude pilote sera publié en 2010, et se composera de deux parties. La première partie réunira les résultats provenant de chaque école afin de résumer les questions communes, les messages et les recommandations de la politique éducative, et elle proposera des moyens d'appliquer la méthodologie dans de futures études. La seconde partie présentera les résultats issus de chaque école, ainsi que des plans, des photos et des citations de personnes participant au projet. Un résumé se rapportant à chaque école fournira une description générale de l'école, ses caractéristiques particulières, les questions importantes en matière de qualité à l'école et les priorités de la politique nationale en ce qui concerne la qualité des espaces éducatifs. Les résultats obtenus par le biais d'outils individuels seront également présentés.

Suite à la publication du rapport final et à l'examen approfondi du processus de mise en œuvre - qui inclura un examen par tous les pays participants du manuel, du Cadre d'évaluation du CELE, des coûts et de l'impact de l'étude - le Conseil des participants du CELE, sous la direction du Groupe CELE d'experts nationaux sur l'évaluation des équipements éducatifs, étudiera la possibilité de développer le projet à plus grande échelle.

Pour en savoir plus, contacter :

Hannah von Ahlefeld

Analyste

Centre de l'OCDE pour des environnements pédagogiques efficaces

2, rue André-Pascal

75116 Paris

France

Hannah.vonAhlefeld@oecd.org

\section{CELE}

Le Secrétariat du CELE remercie les coordinateurs et les équipes de recherche nationaux qui ont participé à la présente étude pour leurs contributions significatives. Brésil : Sheila Ornstein, Professeur en architecture, Urbaniste, Faculté d'Architecture et d'Urbanisme, Université de São Paulo ; Nanci Saraiva Moreira, Architecte, Fondation pour le Développement éducatif ; Rosaria Ono, Architecte, Maître de conférence, Faculté d'Architecture et d'Urbanisme, Université de São Paulo ; Ana Judite Limongi França, Architecte et Urbaniste, étudiante en master, Faculté d'Architecture et d'Urbanisme, Université de São Paulo ; Liliane Cristine Nambu, étudiante de licence, Faculté d'Architecture et d'Urbanisme, Université de São Paulo. Mexique : Carlos Bocanegra Peña, Gerente de Calidad, Formación y Certificación, INIFED ; Jaime de la Garza Reyna, Coordinador para los Asuntos Internacionales, INIFED ; Alfredo Aguilar, Conseiller en Affaires internationales, INIFED. Nouvelle-Zélande : Bruce Sheerin, Analyste politique senior (Immobilier), ministère de l'Éducation. Portugal : Teresa Valsassina Heitor, Architecte, Parque Escolar ; José M.R. Freire da Silva, Architecte, Parque Escolar ; Joana Azevedo, Architecte, Parque Escolar ; Alexandra Alegre, Architecte, Instituto Superior Tecnico ; Susana Catita, Sociologue, Instituto Superior Tecnico. Royaume-Uni : Keith Alexander, Professeur, Centre for Facilities Management, Université de Salford ; Carl Abbott, Directeur de recherche, Université de Salford ; John Lorimer, Capital Programme Director, Manchester City Council ; Bill Scott Watson, Acting Head of School Estates, School Estates, Scottish Government ; Sam Cassels, Design Officer, Education Design Programme, Architecture and Design Scotland ; Hannah Douglas, Programme Officer, Education Design Programme, Architecture and Design Scotland ; et Keith Thomson, Asset Planning, Children and Families Department, City of Edinburgh Council. 


\section{ORGANISATION DE COOPÉRATION ET DE DÉVELOPPEMENT ÉCONOMIQUES}

L'OCDE est un forum unique en son genre où les gouvernements de 30 démocraties œuvrent ensemble pour relever les défis économiques, sociaux et environnementaux que pose la mondialisation. L'OCDE est aussi à l'avant-garde des efforts entrepris pour comprendre les évolutions du monde actuel et les préoccupations qu'elles font naître. Elle aide les gouvernements à faire face à des situations nouvelles en examinant des thèmes tels que le gouvernement d'entreprise, l'économie de l'information et les défis posés par le vieillissement de la population. L'Organisation offre aux gouvernements un cadre leur permettant de comparer leurs expériences en matière de politiques, de chercher des réponses à des problèmes communs, d'identifier les bonnes pratiques et de travailler à la coordination des politiques nationales et internationales.

Les pays membres de l'OCDE sont: l'Allemagne, l'Australie, l'Autriche, la Belgique, le Canada, la Corée, le Danemark, l'Espagne, les États-Unis, la Finlande, la France, la Grèce, la Hongrie, l'Irlande, l'Islande, l'Italie, le Japon, le Luxembourg, le Mexique, la Norvège, la Nouvelle-Zélande, les Pays-Bas, la Pologne, le Portugal, la République slovaque, la République tchèque, le Royaume-Uni, la Suède, la Suisse et la Turquie. La Commission des Communautés européennes participe aux travaux de l'OCDE.

Les Éditions OCDE assurent une large diffusion aux travaux de l'Organisation. Ces derniers comprennent les résultats de l'activité de collecte de statistiques, les travaux de recherche menés sur des questions économiques, sociales et environnementales, ainsi que les conventions, les principes directeurs et les modèles développés par les pays membres.

Cet ouvrage est publié sous la responsabilité du Secrétaire général de l'OCDE. Les opinions et les interprétations exprimées ne reflètent pas nécessairement les vues de l'OCDE ou des gouvernements de ses pays membres.

Les corrigenda des publications de l'OCDE sont disponibles sur : www.oecd.org/editions/corrigenda.

Vous êtes autorisés à copier, télécharger ou imprimer du contenu OCDE pour votre utilisation personnelle. Vous pouvez inclure des extraits des publications, des bases de données et produits multimédia de l'OCDE dans vos documents, présentations, blogs, sites Internet et matériel d'enseignement, sous réserve de faire mention de la source OCDE et du copyright. Les demandes pour usage public ou commercial ou de traduction devront être adressées à rights@oecd.org. Les demandes d'autorisation de photocopier une partie de ce contenu à des fins publiques ou commerciales peuvent être obtenues auprès du Copyright Clearance Center (CCC) info@copyright.com ou du Centre français d'exploitation du droit de copie (CFC) contact@cfcopies.com. 